\title{
Dynamic Absorber Characteristics of Decoupling Coating Covering Underwater Structure
}

\author{
HE Shi-ping \\ College of Power, Naval University of Engineering \\ Hubei Wuhan, China, 430033
}

\author{
LIU Gui-feng \\ Training Department, Naval University of Engineering \\ Hubei Wuhan, China, 430033
}

\begin{abstract}
It is a difficult problem to reduce the low-frequency vibration and radiation noise of underwater structure by decoupling coating. Assuming decoupling coating as viscoelastic fluid, a vibroacoustic model of a simply supported rectangular plate covered by decoupling coating is presented. The model simulates the vibration and acoustic response of the structure which is water-loaded on one side and air backed on the other. The characteristics of vibration and acoustics reduction by the decoupling coating are analyzed. The results show that: 1) For single flexural mode of plate, plate-decoupling coating-water layer is similar to two-freedom degree system, which has two resonant frequencies and an anti-resonant frequency, and dynamic absorber characteristics are introduced by decoupling coating; 2) Dynamic absorber characteristics could be utilized to reduce the low-frequency vibration and underwater radiation noise by selecting suitable parameters of decoupling coating.
\end{abstract}

Keywords-decoupling coating; dynamic absorber; underwater sound radiation; underwater structure

\section{INTRODUCTION}

Noise reduction by means of applying decoupling coating on underwater structures or plates is a commonly used in the marine field [1-3] The aim of the treatment is to isolate the fluid from the vibration of the structures. The decoupling coating should have a low stiffness in comparison to the elastic material of the structures. An ideal condition is that if a vacuum layer is inserted between the outer surface of a submerged structure and the fluid, it will absolutely isolate the transmission of the vibration of the structure, which doesn't radiate underwater sound. However, hydro-compressibility factor of decoupling coating must be controlled. Consequently, there is increased interest in predicting methods of radiation problems from coated structures.

House presented a very simple model of the transmission loss for an infinite panel covered by a decoupling layer and radiating into water[4]. In this model, a closed-form solution of the sound transmission loss is obtained under the assumption that the structure and decoupling coating as fluid-like media. The model predicts a negative insertion loss at a frequency close to the mass-spring mode and a thickness coating mode. Tao meng, Tang Weilin, Fan jun used Hankel transformation and transfer matrix to provide radiation power insertion loss of a point-excited infinite plate covered with multiple compliant layers[1]. The results show that radiation noise increases at low frequencies after the structure is covered with decoupling coating. Yao Xiongliang, Zhang Xu, Qian Dejin etc. carried out a large double cylindrical shell to evaluate the performance of decoupling coating $[5,6]$. The results show that the decoupling coating could decrease the underwater sound radiation obviously, but its capacity is weak when objected to lowfrequency machine exciting.

Another simple description of a compliant coating is the locally reacting model, which assumes that the decoupling coating behaves as evenly distributed massless springs[7-9]. Many authors have used the locally reacting model to describe the vibro-acoustics of underwater structure covered by a decoupling coating. Olivier Foin and Alain Berry presented the vibroacoustic behavior of an elastic, simply supported rectangular plate covered by a locally reacting decoupling layer supporting thickness deformation. The mass-spring mode didn't occur in the results and the decoupling coating didn't increase underwater sound radiation at low frequency.

The objective of this article is to propose a theoretical model to describe the vibration and acoustics behavior of a rectangular, simply supported underwater elastic plate covered by a decoupling coating. The rectangular plate is inserted in an infinite rigid baffle that separates vacuum on one side from water on the other side. The decoupling coating is assumed as fluid-like media. The vibration of the plate, the vibration of the outer surface of the decoupling layer, and the sound radiated in the fluid half-space are investigated when the excitation is a point force applied on the base plate.

\section{THEORECTICAL MODEL}

\section{A. Statement of the problem}

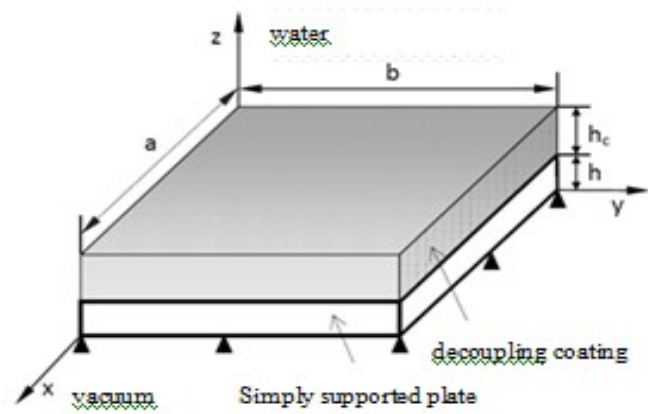

Fig.1 Schematic representation of the simply supported plate covered with a decoupling coating 
The structure is assumed to be a simply supported rectangular plate inserted in an infinite rigid baffle that separates vaccum on one side $(\mathrm{z}<0)$ from water on the other side $(\mathrm{z}>0)$ (Fig. 1$)$. The plate is assumed to conform to the Love-Kirchhoff theory that considers only bending deformation, while the coating is described with the fluid-like model. The plate is excited by a harmonic point force.

\section{B. Description of Equation of Motion}

The equation of motion of the plate, expressed in the frequency domain, is

$$
\tilde{D} \nabla^{4} \tilde{w}_{1}(Q, \omega)-\rho_{S} h \omega^{2} \tilde{w}_{1}(Q, \omega)=\tilde{f}(Q, \omega)-\tilde{\sigma}(Q, \omega)
$$

where w is the angular frequency, $Q$ is a point on the plate, $\tilde{D}$ is the complex bending stiffness of the plate: $\tilde{D}=\tilde{E} h^{3} / 12\left(1-v^{2}\right)$, $\rho_{s}, h, v$ are respectively the density, the thickness, and the Poisson's ratio of the plate. $\tilde{E}$ is the complex Young's modulus, defined by $\tilde{E}=E(1+j \eta), E$ is the real part of Young's modulus, and $\eta$ is the structural loss factor, $\nabla^{4}$ is a partial differential operator: $\nabla^{4}=\frac{\partial^{4}}{\partial x^{4}}+2 \frac{\partial^{4}}{x^{2} y^{2}}+\frac{\partial^{4}}{\partial y^{4}} \cdot \tilde{f}(Q, \omega)$ is the external transverse force per unit area applied to the base plate and $\tilde{\sigma}(Q, \omega)$ is the normal stress in the z-direction applied by the coating on the plate. $\tilde{w}_{1}(Q, \omega)$ is the transverse displacement of the plate.

The transverse displacement of the plate is written in terms of the in-vacuo modes of the plate, which satisfy the simply supported boundary condition (displacement and flexural moment equal to zero):

$$
\tilde{w}_{1}(Q, \omega)=\sum_{m=1}^{\infty} \sum_{n=1}^{\infty} \tilde{a}_{m n}(\omega) W_{m n}(Q)
$$

where $W_{m n}(Q)=\sin \left(m \pi \frac{x}{a}\right) \sin \left(m \pi \frac{y}{b}\right)$.

Generally, the dynamic stiffness of decoupling coating is small, and shear waves have little influence on the vibration and underwater sound radiation of the plate. Here, it is assumed that shear waves don't affect the motion of the plate and decoupling coating. Then, the decoupling coating is assumed as fluid-like media. Applying transfer matrix method, the relation between normal stress $\tilde{\sigma}(Q, \omega)$ in the z-direction applied by the coating on the plate, the acoustic pressure $\tilde{p}(Q)$ of the outer surface of decoupling coating and the transverse displacement $\tilde{w}_{1}(Q, \omega)$ of the plate, the transverse displacement $\tilde{w}_{2}(Q, \omega)$ of the outer surface of decoupling coating could be expressed as

$$
\left(\begin{array}{l}
\tilde{\sigma}(Q) \\
\tilde{p}(Q)
\end{array}\right)=\frac{\rho c \omega}{\sin \left(k h_{c}\right)}\left[\begin{array}{cc}
\cos \left(k h_{c}\right) & -1 \\
1 & \cos \left(k h_{c}\right)
\end{array}\right]\left(\begin{array}{c}
\tilde{w}_{1}(Q) \\
\tilde{w}_{2}(Q)
\end{array}\right)
$$

where $\rho, h_{c}, c$ are respectively the density, the thickness, and the sound velocity of the decoupling coating. $k$ is the wave number of longitudinal wave of the decoupling coating $k=\omega / c$.

Assuming the complex Young's modulus of decoupling coating is $\tilde{E}_{c}=E_{c}\left(1+j \eta_{c}\right), E_{c}$ is the real part of Young's modulus, and $\eta_{c}$ is the loss factor, the sound velocity of longitudinal wave can be expressed as

$$
c=\sqrt{\tilde{E}_{c} / \rho}
$$

The acoustic pressure in the fluid is governed by the Helmholtz equation,

$$
\nabla^{2} \tilde{p}(M)+k_{0}^{2} \tilde{p}(M)=0 \quad\left(k_{0}=\omega / c_{0}\right)
$$

where $M$ is a point in the fluid space, $c_{0}$ is the velocity of sound in the fluid.

The equation of continuity of the structural and acoustic normal acceleration on the outer surface of the decoupling material is

$$
\frac{\partial \tilde{p}(M)}{\partial z}=\rho_{0} \omega^{2} \tilde{w}_{2}(Q)
$$

where $\rho_{0}$ is the density of the fluid.

The acoustic pressure on the surface of the coating is expressed as the Rayleigh integral[10],

$$
\tilde{p}(Q)=-\iint\left[\frac{\partial \tilde{p}(M)}{\partial n_{M}} G(Q, M)\right] d s_{M}
$$

where $s$ is the surface of the decoupling coating, points $Q$ and $M$ are on the surface of the decoupling coating, $G(Q, M)$ is acoustic Green's function,

$$
G(Q, M)=G\left(x, y, x^{\prime}, y^{\prime}\right)=\frac{e^{-j k_{0} \sqrt{\left(x-x^{\prime}\right)^{2}+\left(y-y^{\prime}\right)^{2}}}}{2 \pi \sqrt{\left(x-x^{\prime}\right)^{2}+\left(y-y^{\prime}\right)^{2}}}
$$

The transverse displacement of the outer surface of the decoupling coating is written in terms of the in-vacuo modes of the plate:

$$
\tilde{w}_{2}(Q, \omega)=\sum_{m=1}^{\infty} \sum_{n=1}^{\infty} \tilde{b}_{m n}(\omega) W_{m n}(Q)
$$

Using Eq.(3),(6) and (9), Eq.(7) becomes

$$
\begin{aligned}
& -\iint_{s}\left[\rho_{0} \omega^{2} \tilde{w}_{2}(Q) G(Q, M)\right] d s_{M} \\
& =\frac{\rho c \omega}{\sin \left(k h_{c}\right)}\left[\tilde{w}_{1}(Q)-\cos \left(k h_{c}\right) \tilde{w}_{2}(Q)\right]
\end{aligned}
$$

Using the orthogonal properties of the natural modes, Equations (1) and (10) are two systems of coupled equations in terms of the modal unknows of the problems: $\tilde{a}_{m n}$ and $\tilde{b}_{m n}$ $\forall(m, n) \in[1, \cdots N]^{2}$, and be written in 


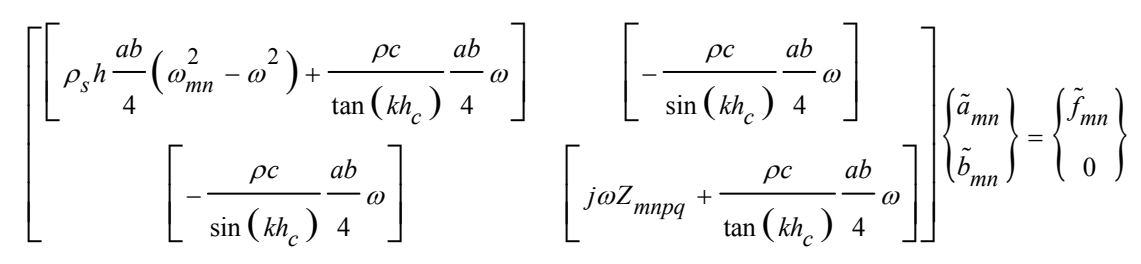

where $Z_{\text {mnpq }}$ are the coefficients of the radiation impedance matrix defined by:

$$
Z_{m m p q}=j \rho_{0} \omega \iint_{S} \iint_{S} W_{p q}(M) G(Q, M) W_{m n}(Q) d s_{M} d s_{Q}
$$

$\omega_{m n}$ is the in-vacuo natural angular frequencies of the plate:

$$
\omega_{m n}=\sqrt{\frac{D}{\rho_{s} h}}\left(\left(\frac{m \pi}{a}\right)^{2}+\left(\frac{n \pi}{b}\right)^{2}\right)
$$

$\tilde{f}_{m n}$ is the generalized vector of forces:

$$
\tilde{f}_{m n}=F_{0} \sin \left(m \pi \frac{x_{0}}{a}\right) \sin \left(n \pi \frac{y_{0}}{b}\right)
$$

where $F_{0}$ is the magnitude of the force, and $x_{0}$ and $y_{0}$ are the coordinates of the excitation point.

To solve the problem, it is needed to truncate the infinite series $(\mathrm{m}, \mathrm{n}, \mathrm{p}, \mathrm{q})$ to a finite order N. Equation (11) becomes a matrix system with dimensions $2 N^{2} \times 2 N^{2}$. The unknown coefficients $\tilde{a}_{m n}$ and $\tilde{b}_{m n}$ can be calculated, which allows vibration velocity and sound radiation power to be calculated.

The mean square velocity of the base plate is defined by

$$
\left\langle v^{2}\right\rangle=\frac{\omega^{2}}{2 s} \iint_{s} \tilde{w}_{1}(Q) \tilde{w}_{1}^{*}(Q) d s_{Q}
$$

where * designates the complex conjugate. Using the expansion of $\tilde{w}_{1}$ over the orthogonal in vacuo modes of the plate, one gets

$$
\left\langle v_{1}^{2}\right\rangle=\frac{\omega^{2}}{8} \sum_{m=1}^{N} \sum_{n=1}^{N}\left|\tilde{a}_{m n}\right|^{2}
$$

Similarly, the mean square velocity of the outer surface of the decoupling coating is

$$
\left\langle v_{2}^{2}\right\rangle=\frac{\omega^{2}}{8} \sum_{m=1}^{N} \sum_{n=1}^{N}\left|\tilde{b}_{m n}\right|^{2}
$$

The radiated sound power in the fluid is the integration of the active acoustic intensity normal to the decoupling coating,

$$
\begin{aligned}
W & =\frac{1}{2} \operatorname{Re}\left[\iint_{s} \tilde{P}(Q)(-j \omega) \tilde{w}_{2}^{*}(Q) d s_{Q}\right] \\
& =\frac{\omega^{2}}{2} \sum_{m=1}^{N} \sum_{n=1}^{N} \tilde{b}_{m n} \operatorname{Re}\left[Z_{m n p q}\right] \tilde{b}_{p q}^{*}
\end{aligned}
$$

The level of radiated power and quadratic velocity are defined as:

$$
L_{v}=10 \log \left(v^{2} / v_{0}^{2}\right) \quad v_{0}=5 \times 10^{-8} \mathrm{~m} / \mathrm{s}
$$

$$
L_{w}=10 \log \left(W / W_{0}\right) \quad W_{0}=10^{-12} \text { Watt }
$$

\section{DYNAMIC ABSORBER CHARACTERISTICS}

The plate studied is immersed in water $\left(\rho_{0}=1000 \mathrm{~kg} / \mathrm{m}^{3}\right)$, $c_{0}=1500 \mathrm{~m} / \mathrm{s}$ ) and is $780 \mathrm{~mm}$ long, $580 \mathrm{~mm}$ wide, and $24 \mathrm{~mm}$ thick. It is made of steel with a loss factor $\eta$ equal to 0.005 . The thickness of decoupling coating is $h_{c}=30 \mathrm{~mm}$, the complex Young's modulus is $E_{c}=5 \times 10^{6}(1+0.1 i) \mathrm{N} / \mathrm{m}^{2}$, and the density $\rho=1100 \mathrm{~kg} / \mathrm{m}^{3}$. The mechanical excitation is a z-direction point force applied at $1 / 4$ of length and width of the plate with a amplitude of $1 \mathrm{~N}$.

Figures 2 and 3 deal, respectively, with the quadratic velocity, and the radiated power of the plate with and without decoupling coating. The $\mathrm{x}$-coordinate is the logarithm of the frequency. The quadratic velocity of the plate presents different behaviors depending on the frequency. In the low frequency domain $(\mathrm{f}<70 \mathrm{~Hz})$, the coating is ineffective for modifying the level. In the medium frequency range $(110 \mathrm{~Hz}<\mathrm{f}<220 \mathrm{~Hz})$, the vibration level is reduced by the decoupling coating. Above $220 \mathrm{~Hz}$, the location of vibration peak frequency changes and the plate vibration increases in tendency. The radiated power is generally reduced by the decoupling coating, except below $120 \mathrm{~Hz}$.

Generally, the decoupling coating weakens the coupling between the plate and the fluid. This means the decrease of fluid added mass which leads to that the resonant frequency of plate shift to higher frequency. However, the first peak frequency (about $100 \mathrm{~Hz}$ ) of quadratic velocity and sound radiation of the plate with decoupling coating is lower than the first peak frequency (about $180 \mathrm{~Hz}$ ) without decoupling coating.

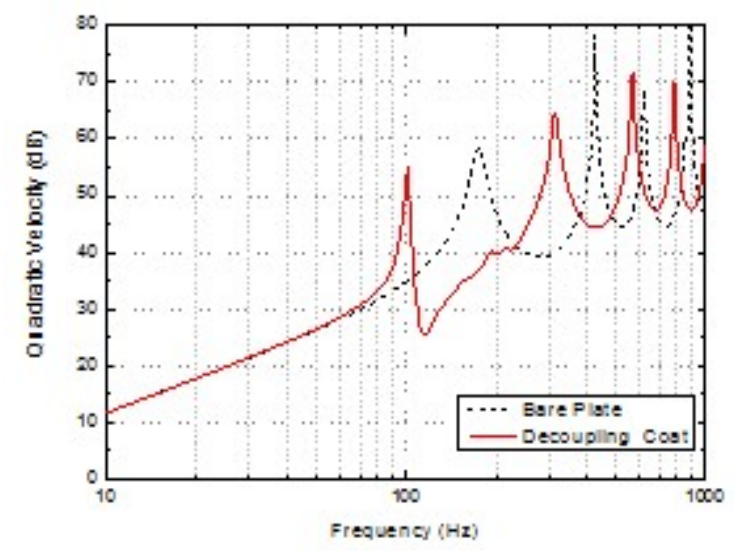

Fig.2 Quadratic velocity of the plate; solid line: covered with decoupling coating; dash line: bare plate 


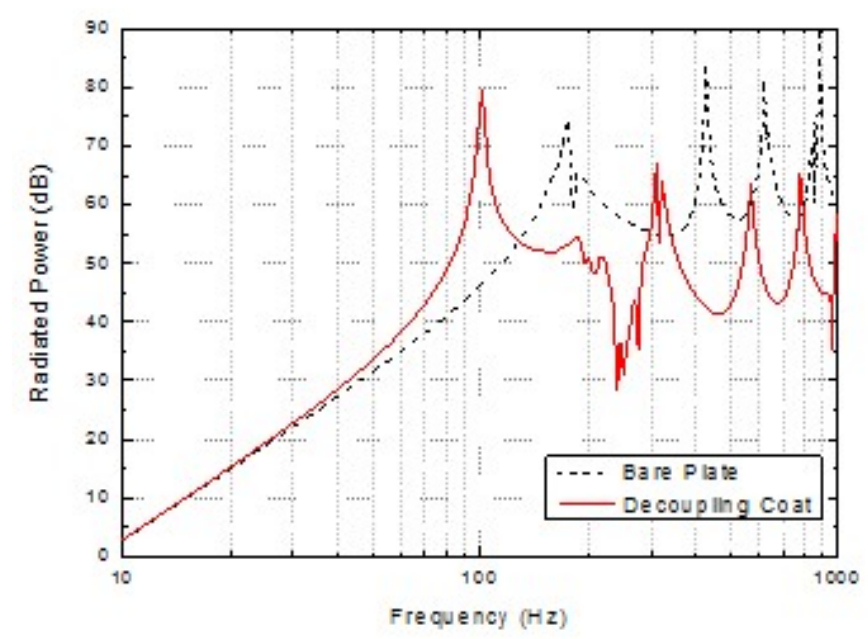

Fig.3 Radiated power of the plate; solid line: covered with decoupling coating; dash line: bare plate

Decoupling coating is used to isolate the fluid from the vibration of the plate. For single mode of the plate, if the vibration of the plate is assumed as vibration input, the "decoupling coating-fluid system" can be looked as a massdamp-spring system. The mean square velocity ratio between plate and outer surface of decoupling coating is given in Fig.4 The results show that vibration of outer surface of decoupling coating increases below 300Hz. However, from Fig. 2 and Fig.3, there exist a frequency domain within $300 \mathrm{~Hz}$ at which the vibration and sound radiation decrease.

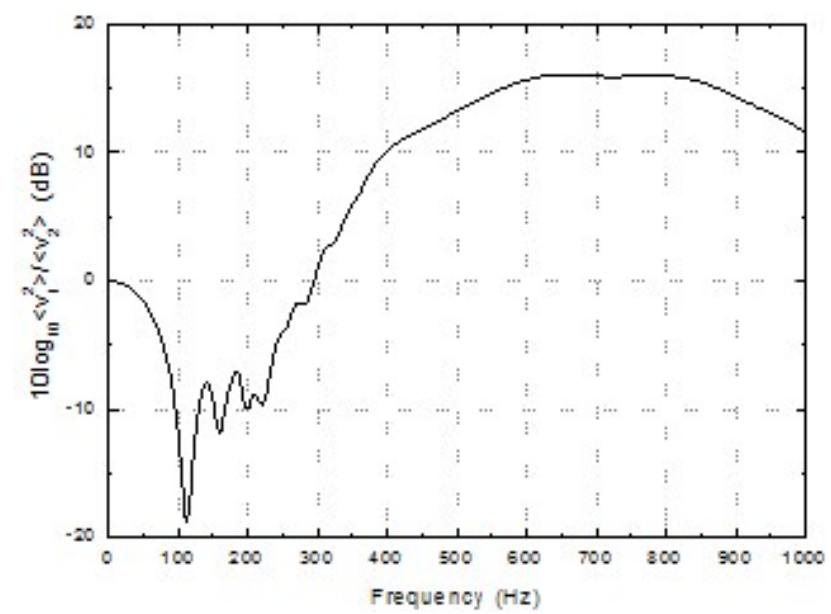

Fig. 4 Mean square velocity ratio between the plate and the outer surface of decoupling coating

To further analyze the phenomenon, let us introduce a modal decomposition, neglect fluid modal cross coupling, and assume $k h_{c} \rightarrow 0$, equations (11) becomes:

$$
\left\{\begin{array}{c}
\left(\rho_{s} h \frac{a b}{4}\left(\omega_{m n}^{2}-\omega^{2}\right)+\frac{E_{c}}{h_{c}}\right) \tilde{a}_{m n}-\frac{E_{c}}{h_{c}} \tilde{b}_{m n}=\tilde{f}_{m n} \\
-\frac{E_{c}}{h_{c}} \tilde{a}_{m n}+\left(\frac{E_{c}}{h_{c}}+j \omega Z_{m n p q}\right) \tilde{b}_{m n}=0
\end{array}\right.
$$

These equations are those of the two degree of freedom system and describe the basic behavior of modes of a plate covered with a decoupling coating. Such a vibratory system is known to have two resonance angular frequencies.

$$
\omega_{n}^{2}=\frac{\omega_{a}^{2}(1+\mu)+\omega_{m n}^{2}}{2} \pm \sqrt{\left(\frac{\omega_{a}^{2}(1+\mu)-\omega_{m n}^{2}}{2}\right)+\omega_{a}^{2} \omega_{m n}^{2} \mu}
$$

where $\omega_{a}$ is the resonant angular frequency of coating-fluid added mass system.

$$
\omega_{a}^{2}=\left(E_{c} / h_{c}\right) / M_{f}
$$

$\mu=M_{f} / \rho_{s} h, M_{f}$ is the fluid added mass of infinite plate of same thickness and material as the plate.

According to the reference[10], assuming thickness of a fluid added mass layer to join vibrations is roughly $1 / 6$ of a flexural wavelength in the plate, the ratio of fluid added mass to plate mass can be expressed as:

$$
\mu^{5}+\mu^{4}-\frac{1}{4 \pi^{2}}\left(\frac{\rho_{0}}{\rho_{s}}\right)^{4}\left(\frac{\lambda_{b}}{h}\right)^{4}=0
$$

where $\lambda_{b}$ is the wavelength of flexural wave. For the two degree of freedom system, one resonant frequency $\omega_{n 1}$ is smaller than $\omega_{a}$, another resonant frequency $\omega_{n 2}$ is greater than $\omega_{a}$.

Actually, the coating-fluid system can be taken as a dynamic absorber. When the resonant frequancy $\omega_{a}$ of the coating-fluid system is close to the resonant frequency $\omega_{m n}$, effect of anti-resonance is very strong. This interpret the reason of vibration and noise reduction within $[110,220] \mathrm{Hz}$.

\section{CONCLUSION}

Assuming decoupling coating as viscoelastic fluid, a vibration and acoustics model of a simply supported rectangular plate covered by decoupling coating is presented. The model simulates the vibration and acoustic response of the structure which is water-loaded on one side and air backed on the other. The characteristics of vibration and acoustics reduction by the decoupling coating are analyzed. The results show that: 1) For single flexural mode of plate, platedecoupling coating-water layer is similar to two-freedom degree system, which has two resonant frequencies and an antiresonant frequency, and dynamic absorber characteristics are introduced by decoupling coating; 2) Dynamic absorber characteristics could be utilized to reduce the low-frequency 
vibration and underwater radiation noise by selecting suitable parameters of decoupling coating.

\section{REFERENCES}

[1] Tao meng, Tang Weilin, Fan Jun. "Mechanism analysis of noise reduction by compliant decoupling layers," Journal of Ship Mechanics, Vol.14(4), pp. 220-225. In Chinese

[2] Tao meng, Fan Jun, Tang Weilin. "The characteristics of sound radiation from a cylindrical shell coated with multiple compliant layers," ACTA ACUSTICA, 2008,Vol.33(3), pp. 421-429. In Chinese

[3] Miao Xuhong, Wang Renqian, Gu lei. "Numerical analysis on the sound insulation performance of decoupling material," Journal of Ship Mechanics, 2005,Vol.9(5), pp. 125-131. In Chinese

[4] House,J.R.C. "Considerations for underwater decoupling treatment," Proc.I.O.A, 1991, 13:166-173.

[5] Yao Xiongliang, Zhang Aman, Qian Dejin, Pang Fuzhen. "On effect of laying regious of uncoupled anechoic material on sound radiation of double cylindrical shell," Journal of naval university of engineering, 2008, 20(2):33-37. In Chinese

[6] Yao Xiongliang, Zhang Xu, Qian Dejin, Zhang Aman. "Experiment research of acoustics performance of separate-sound and decoupled material," Transducer and microsystem technologies, 2008, 27(7):31-33. In Chinese

[7] Crighton, D.G. "Aspects of the reflexion and free wave properties of a composite panel under fluid loading," Journal of sound and vibration, 1979, 64(4):467-474.

[8] Maidanik,G. "Modification caused by compliant layers and blankets in the pressure field induced on a boundary," Trans. ASME. J. Vib. Acoust. Stress. Reliab. Des. 1984, 106(3):369-375.

[9] Olivier F. and A. Berry. "Acoustic radiation from an elastic baffled rectangular plate covered by a decoupling coating and immersed in a heavy acoustic fluid," J.Acoust. Soc. Am, 2000, 107(5):2501-2510.

[10] S.Nikiforov, translated from Russian by Alexander Katalov and Dimitri Donskoy. Acoustic design of naval structures. USA, Naval Surface Warfare Center, 2005. 